\title{
The Generating Algorithm and Case Study for the Spectral Reflectance Images of Ground Features
}

\author{
Zhaolu Zhang, ${ }^{1, *}$, Yunjun $\mathrm{Yao}^{2}$, Haitao $\mathrm{Cao}^{3}$ \\ ${ }^{1}$ School of Mineral Resources and Environmental Engineering, Shandong University of Technology, Zibo Shandong, China \\ ${ }^{2}$ State Key Laboratory of Remote Sensing Science, College of Global Change and Earth System Science, Beijing Normal University, Beijing, \\ China \\ ${ }^{3}$ Department of Geological and Mining Engineering and Sciences, Michigan Technological University, Houghton, USA
}

Email address:

shanguf@qq.com (Zhaolu Zhang)

${ }^{*}$ Corresponding author

\section{To cite this article:}

Zhaolu Zhang, Yunjun Yao, Haitao Cao. The Generating Algorithm and Case Study for the Spectral Reflectance Images of Ground Features. American Journal of Remote Sensing. Vol. 4, No. 2, 2016, pp. 9-12. doi: 10.11648/j.ajrs.20160402.11

Received: March 14, 2016; Accepted: March 28, 2016; Published: April 16, 2016

\begin{abstract}
The paper got the data of spectral reflectance of ground features from field surveying by using field spectroradiometer. The spatial distribution information of the ground features was obtained from the land-use map. Based on above mentioned, the generating algorithm of spectral reflectance image of ground features was developed by Modeler module of ERDAS Imaging software. The four bands were selected as example image bands, including the blue band (0.45-0.52im), the green band $(0.52-0.60 \mathrm{im})$, the red band $(0.63-0.69 \mathrm{im})$ and the infrared band $(0.76-0.90 \mathrm{im})$. The four band images with real geographical coordinates were generated from the spectral reflectance of ground features. In order to present the following images, the true color and the standard false color images were merged with four individual band images. By using the field spectroradiometer, relatively simple compared with hyperspectral imaging radiometer, the similar spectral reflectance images of ground features could be obtained with the secondary developed generating algorithm on the ERDAS Imaging software platform. Through the analysis of the spectral reflectance images of ground features, we can prove that the generated images are close to the real land scenes. Therefore, this paper provides a new idea and a new method for the first step of simulating remote sensing images with real geographic coordinates. Finally, the authors prefer to explain that further studies should be developed in two aspects. One issue is how to describe the spatial distributing information of ground features more accurately, and the other is how to differentiate the same class ground features with different spectral reflectance. Based on above, further more studies should include the effect of topographic factors on the spectral reflectance of ground features.
\end{abstract}

Keywords: The Spectral Reflectance Image of Ground Features, Generating Algorithm, ERDAS Imaging Software, Field Spectro-radiometer, Merged Image

\section{Introduction}

The spectral reflectance, an inherent property of ground features, is the ratio of the reflective radiation to the incident radiation of a ground feature. And it is also the fundament for remote sensing work. The spectral reflectance of various bands of observed ground features can be measured with a field spectro-radiometer. Thereby the spectral reflectance curves of ground features can be drawn [1]. After obtaining the spectral reflectance of every geo-spatial point, It becomes the key process how to generate the spatial spectral reflectance images from these spectral reflectance data. Accordingly, the generating algorithm of the spectral reflectance image of ground features is critical for solving the problem as we discussed above, and is also the premise to perform simulation for the remote sensing image [2]. Based on the process of acquiring the remote sensing image, the simulation of RS image usually consists of three steps. Respectively, they are the generation of the image of ground scenes, the simulation of the process of atmospheric radiative transfer and the simulation of sensor optical system as well [3, 4]. Particularly, it is noteworthy to point out that the high 
quality of the spectral reflectance image is the prerequisite for the derivation of remote sensing image with higher resolution. In this paper, the Modeler module of ERDAS Imaging software is performed as the technical platform and it provides a practical way to generate the algorithm for the spectral reflectance image of ground features [5, 6]. A corner of the Shandong University of Technology is selected as the research zone with the topography effect excluded.

\section{The Acquisition and Processing of the Spectral Reflectance of Ground Features}

\subsection{The Field Survey of the Spectral Reflectance of Ground Features}

The ISI921VF-128 spectroradiometer with eight nanometer resolution, produced by Hefei Intelligent Scientific Instruments Technology Co., Ltd, is applied to measure the spectral reflectance of ground features in field. In this case, we focus on reflective radiation ranging from visible light to near-infrared band $(0.38-1.08 \mu \mathrm{m})$. Based on the bidirectional reflective factor theory [7, 8], three types of ground features composed of concrete pavement, grassland and bush area as well, are involved in the spectral reflectance survey. Ten typical sample points are chosen for each type of ground features, and then every point is surveyed three to five times. Finally, we take the average surveying data to draw the spectral reflectance curves [9].

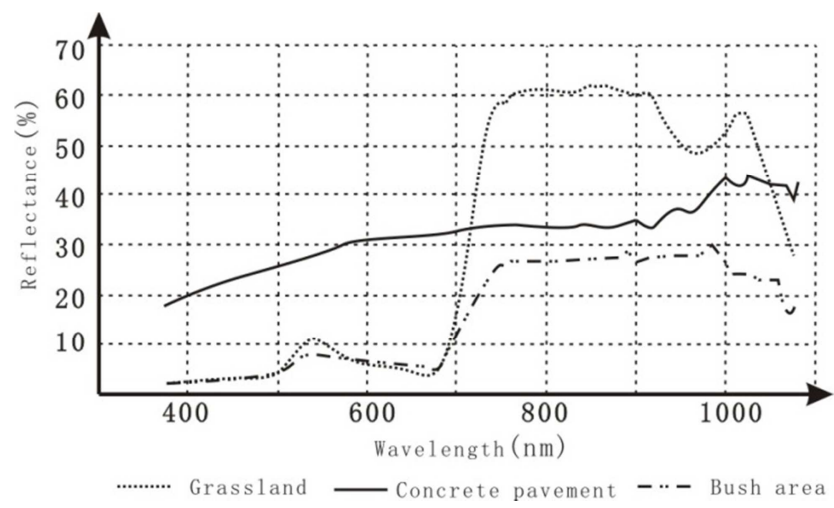

Fig. 1. The spectral reflectance curve of ground features through field surveying

\subsection{Data Preparation}

The wavebands of the spectral reflectance image of ground features are designed to correspond with the bands of TM 1-4 of land resources satellite, namely, the blue band $(0.45-0.52 \mu \mathrm{m})$, the green band $(0.52-0.60 \mu \mathrm{m})$, the red band $(0.63-0.69 \mu \mathrm{m})$ and the infrared band $(0.76-0.90 \mu \mathrm{m})$. Because the ranges of four bands are wider than that of the spectral resolution of spectroradiometer $(8 \mathrm{~nm})$, we adopt the average measured value of each band of ground features as its reflectance value so as to generate the spectral reflectance image of ground features.
Table 1. The spectral reflectance values of ground features in different bands.

\begin{tabular}{lllll}
\hline & Blue band & Green band & Red band & Infrared \\
\hline Grassland & 3.83 & 8.79 & 5.03 & 60.69 \\
$\begin{array}{l}\text { Concrete } \\
\text { pavement }\end{array}$ & 24.05 & 28.83 & 31.33 & 33.17 \\
Bush area & 3.51 & 7.04 & 5.76 & 26.92 \\
\hline
\end{tabular}

The spatial resolution of the spectral reflectance image of ground features is set as $30 \times 30 \mathrm{~m}^{2}$. The spatial distributing information of ground features originate from the land-use map which is turned into a raster map.

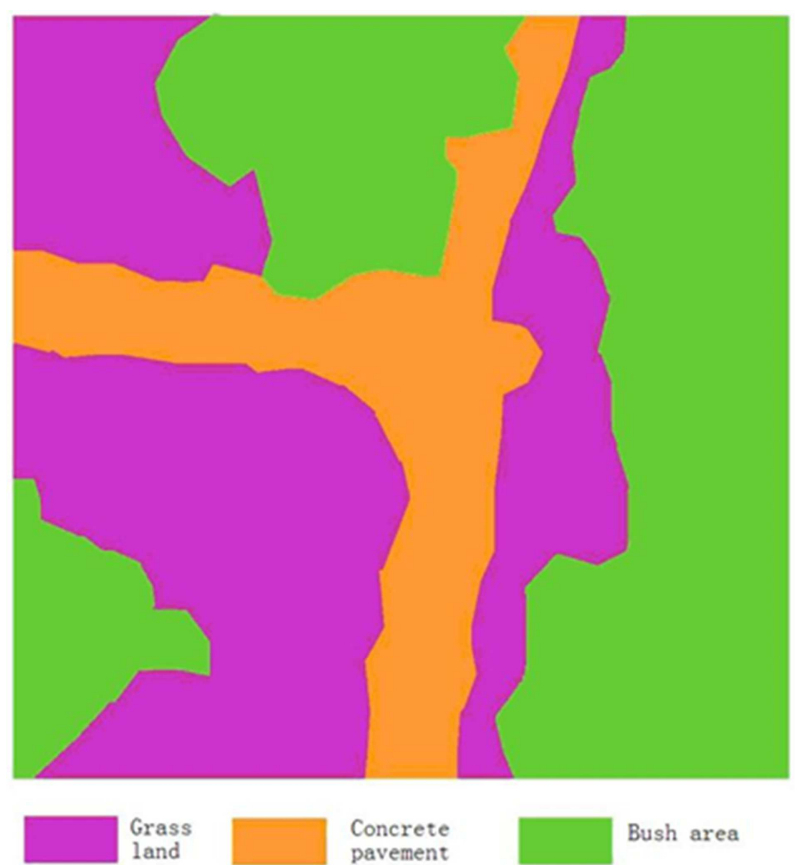

Fig. 2. The spatial distribution raster map of ground features in researching zone.

\section{The Generating Algorithm and Case Study}

\subsection{Methodology}

The detailed algorithm is realized by graphic modeling which is one function of the Modeler module of ERDAS Imaging software. The input data comprise two parts. One is the spatial distribution raster map, which is regarded as the information source of ground features; the other is the spectral reflectance values, which is showed in table 1 . The next step is to define a function to assign the spectral reflectance value for every ground feature respectively. The blue band is selected as an example, which is showed in formula 1.

$$
\begin{aligned}
& \text { Conditional (grassland 3.83, concrete } \\
& \text { pavement 24.05, bush area } 3.51 \text { ) }
\end{aligned}
$$

Similarly, the images of green band, red band and infrared band are generated through the same algorithm. The whole technical steps are illustrated in Figure 3. 


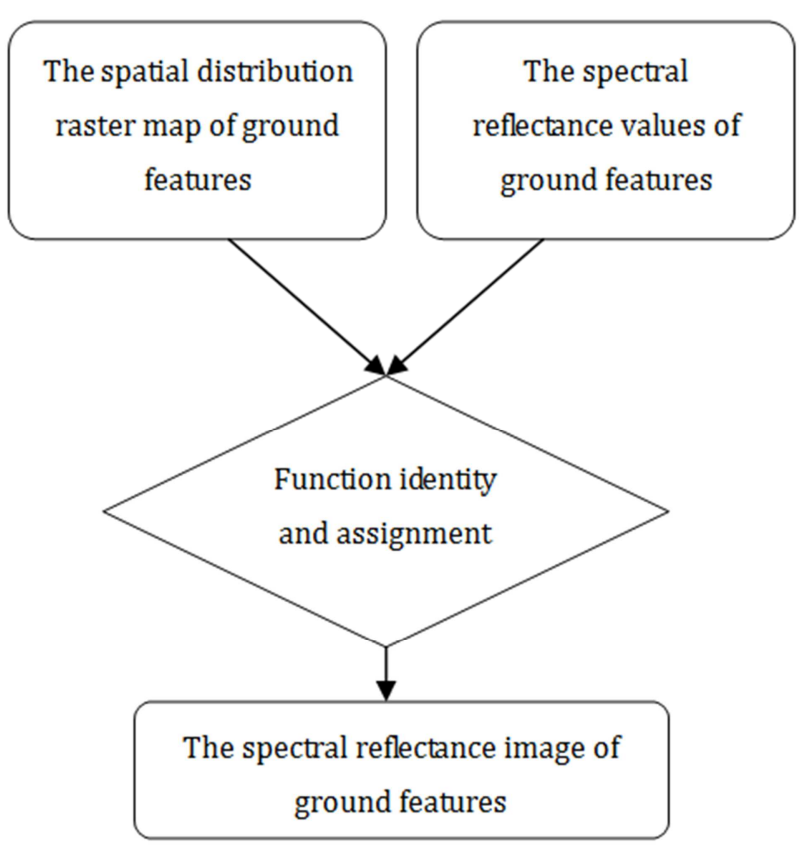

Fig. 3. The flow chart of generating the spectral reflectance map of ground features.

\subsection{Analyses on the Experimental Results}

The multi-spectral reflectance images of ground features, consisting of blue, green, red and infrared band respectively, are generated through the algorithm we discussed above in the paper. In order to enhance the display effect of the spectral reflectance image, both the true color composite image and the standard false color composite image are synthesized and utilized for visual interpretation.

In the blue band image, the spectral reflectance of grassland is 3.83 , bush area 3.51 , and concrete pavement 24.05. Compared with the concrete pavement, the spectral reflectance of both bush area and grassland are much lower than that of concrete pavement. Therefore, in the spectral reflectance image of blue band, the concrete pavement displays white, but grassland and bush area show black color (Figure 4-a).

In the green band image, the spectral reflectance of grassland is 8.79 , bush area 7.04, and concrete pavement 28.83. Compared with the concrete pavement, both grassland and bush area show much lower reflectance value, but the difference is narrowing. Additionally, the spectral reflectance of grassland is a little higher than that of bush area. Consequently, the concrete pavement displays white, while grassland shows gray and bush area is black (Figure 4-b).

In the red band image, the spectral reflectance of grassland is 5.03, bush area 5.76 and concrete pavement 31.33. Compared with the concrete pavement, the spectral reflectance of grassland and bush area are much lower. In addition, the spectral reflectance of bush area is a little higher than that of grassland. According to the derived image of red band, the concrete pavement demonstrates white, while grassland is black and bush area shows deep gray (Figure 4-c).
In the infrared band image, the spectral reflectance of grassland is 60.69 , bush area 26.92 , concrete pavement 33.17 . The grassland indicates the highest spectral reflectance followed by concrete pavement and the bush area. Accordingly, the three colors, white, gray and black respectively represent grassland, concrete pavement and bush area in the spectral reflectance image of infrared band (Figure 4-d).

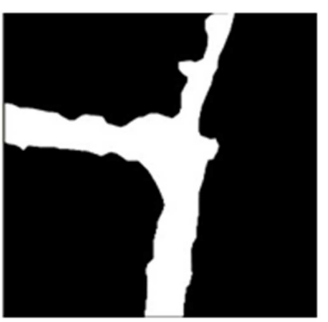

(a). blue band

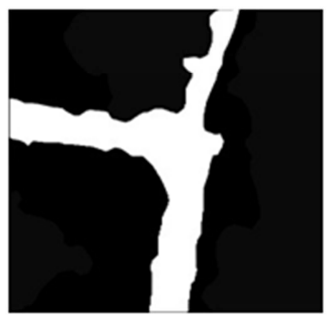

(c). red band

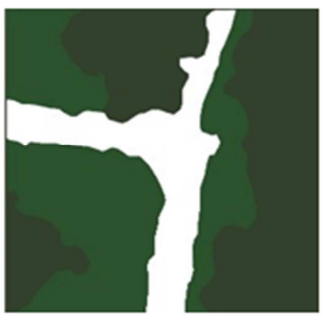

(e). True color

Grass land

Bush area

Concrete pavement

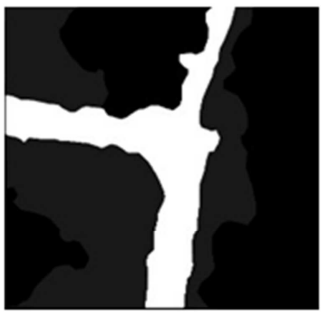

(b). green band

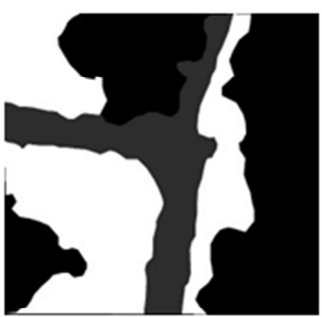

(d). infrared band

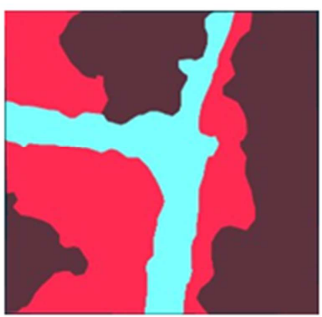

(f). Standard false color

Grass land

Bush area

Concrete pavement
Fig. 4. The spectral reflectance images of ground features generated by the algorithm.

Based on the additive color law [10], the four spectral reflectance images of ground features are synthesized to the true color composite image and the standard false color composite image. In the true color composite image, grassland shows green color, while bush area presents dark green and concrete pavement indicates white. In this way, the true color composite image resembles what would be observed by human naked eyes. In terms of the standard false color composite image, grassland shows aubergine, while bush area presents dark red and concrete pavement appears light cyan. In this case, all the ground features in the standard false color composite image resemble the appearance of the TM standard false color image (Figure 4-f). 


\section{Conclusion and Prospect}

A new algorithm for generating the spectral reflectance image of ground features has been presented in this paper. It has been developed based on the Modeler module of ERDAS Imaging software. The algorithm needs two input data. One is the spatial distribution raster map, which is regarded as the information source of ground features; the other is the spectral reflectance data, which is surveyed in field by spectroradiometer. The spectral reflectance image of ground features could be obtained through the auto process which is based on the algorithm. By using the relatively simple field spectroradiometer rather than a hyperspectral radiometer, the similar spectral reflectance image with high quality can be obtained through the method discussed above. According to the analyses of the spectral reflectance images of ground features in this paper, the generated images are close to the real land scene. Therefore, the case study provides a new idea and a new method for the first step of simulating remote sensing image with real geographic coordinates. Meanwhile, two aspects need to be developed and further analyzed. One issue is to describe the spatial distributing information of ground features more accurately, and the other is to enhance the resolution of the ground features which belong to the same class with different spectral reflectance. Additionally, if the effect of the topographic relief should be taken into consideration, the derived spectral reflectance image would be more close to the real land scene.

\section{Acknowledgements}

This work was supported by the National Science Foundation of China under Grant number 41201331; the Outstanding Young Teacher Supporting Plan of Shandong University of Technology under Grant.

\section{References}

[1] Ting He, Ye Cheng and Jing Wang. 2002. "The method and technology of surveying ground feature spectrum in the wild." Land Science of China, 16(5): 30-36.

[2] Zhaolu, Zhang. 2010. "The improvement of experimental scheme for the ground feature spectral reflective ratio for the field surveying." Science of Surveying and Mapping, 35(5): 176-177(175).

[3] Guanter, L., Segl K and Kaufmann H. 2009. "Simulation of Optical Remote-Sensing Scenes With Application to the EnMAP Hyperspectral Mission." IEEE Transactions on Geoscience and Remote Sensing, 47(7 Part 2): 2340-2351.

[4] Fang Chen, Zheng Niu and Chuhong Liao.2006. "The Analysis of method and application of simulating technology of remote sensing." Geo-information Science, 8(3): 114-118.

[5] Ayman Rashad Elshehaby, Lamyaa Gamal El-deen Taha. 2009. "A new expert system module for building detection in urban areas using spectral information and LIDAR data." Applied Geomatics, 1(4): 97-110.

[6] Qinqin Sun, Jianjun Tan and Yonghang Xu. 2009. "An ERDAS image processing method for retrieving LST and describing urban heat evolution: a case study in the Pearl River Delta Region in South China." Environmental Earth Sciences, 59(5): 1047-1055.

[7] Anxin Mei, Wanglu Peng and Qiming Qin. 2001. The Outline of Remote Sensing. Beijing: High Education Press.

[8] Ai-hua Wang, P. F. Hsu and Jiu-ju Cai. 2010. "Modeling bidirectional reflection distribution function of microscale random rough surfaces." Journal of Central South University of Technology, 17(2): 228-234.

[9] Huiping Liu, Qiming Qin and Wanglu Peng. 2001. The Tutorial of remote sensing practice. Beijing: High Education Press.

[10] Guoan Tang, Youshun Zhang and Yongmei Liu. 2004. The process of Remote sensing Digital Image. Beijing: High Education Press. 